\title{
Association between multiple sclerosis disease severity and adherence to disease-modifying therapies
}

\author{
Adam Burkhard, PharmD; Joshua Toliver, PharmD, PhD; and Karen Rascati, PhD
}

\section{What is already known about this subject}

- Multiple sclerosis (MS) is a chronic autoimmune disorder leading to demyelination of neurons throughout the central nervous system.

- Disease modifying therapies (DMTs) can delay disease progression, but barriers to DMT adherence such as perceived lack of efficacy, issues with injections, and adverse events can lead to poor adherence and increased cost burden to patients and plan sponsors.

- Although limited evidence is available, demographic parameters such as age, sex, DMT type, and comorbidities are associated with adherence rates.

\section{What this study adds}

- This retrospective study evaluated adherence to DMTs for the treatment of MS specific to the Medicare population.

- Disease severity is rarely considered when evaluating risk factors for poor adherence; however, patients with a high MS disease severity score were $53 \%$ less likely to be adherent compared to those patients with a low MS disease severity score.

- MS disease severity should be considered when evaluating risk of low adherence.

\section{ABSTRACT}

BACKGROUND: For multiple sclerosis (MS) patients taking disease-modifying therapies (DMTs), adherence to treatment is a key component of achieving beneficial outcomes, such as delayed disease progression and the reduction and prevention of symptoms and relapses.

OBJECTIVES: The aim of this study was to assess the impact of a claims-based measure of MS disease severity on DMT adherence in a one-year study period.

METHODS: Patients were identified from Humana Medicare Advantage claims data from January 1, 2013 to December 31, 2015. Patients over the age of 18 with at least
12 months of continuous enrollment and $>1$ outpatient MS visit with DMT use prior to the index date were included. Patients who switched DMT type (oral, platform, IV) during the study period were excluded. Medication possession ratios (MPR) for DMTs were calculated from pharmacy and medical claims over 12 months of claims data, and a previously developed claims algorithm was used to determine MS disease severity. Patients with MPRs of 0.8 or higher were considered adherent to DMT treatment. Multivariable logistic regression was used to evaluate the association of MS disease severity, gender, DMT type, and age category with DMT adherence.

RESULTS: The study population of 3,347 patients had an average MPR of 84.7 (75\%

\section{Author affiliations \\ Adam Burkhard, PharmD; Joshua Toliver, PharmD, PhD; Karen Rascati, PhD; The University of Texas at Austin, Austin, TX.}

\section{AUTHOR CORRESPONDENCE:}

Karen Rascati, 512.471.1637;

krascati@mail.utexas.edu

J Manag Care Spec Pharm 2021;27(7):915-23

Copyright (C2021, Academy of Managed Care Pharmacy. All rights reserved.

were classified as adherent). Multivariable logistic analysis demonstrated that compared to the 18-45 age group, the $46-64$ and $65+$ age groups were 1.33 (OR: 1.33 [95\% Cl 1.081.64]) and 1.55 (OR: 1.55, [95\% Cl 1.18-2.05]) times more likely to be adherent. Patients with a high level of MS disease severity were 53\% (OR: 0.47, [95\% Cl 0.36-0.62]) less likely to be adherent compared to those with low MS disease severity. No significant difference was identified for gender or DMT type (oral, platform, or IV).

CONCLUSIONS: Increased age and lower MS disease severity were associated with better DMT adherence. MS disease severity should be considered when assessing risk for low DMT adherence. 
Multiple sclerosis (MS) is a chronic autoimmune disorder of the central nervous system (CNS) resulting in the demyelination of neurons throughout the brain and spinal cord. ${ }^{1}$ Although no cure for MS exists, disease modifying therapies (DMTs) are often used to delay disease progression and reduce or prevent MS symptoms..$^{2-5}$ As with many chronic diseases, management of MS is complicated by patient levels of DMT adherence. ${ }^{6}$ Poor adherence negatively impacts effectiveness of DMTs and increases cost burden on health plans, plan sponsors, and the healthcare system..$^{711}$ While there are many barriers to DMT adherence in MS patients, issues with injections, perceived lack of effectiveness, and adverse events have been identified as the most common. ${ }^{12}$

The fear of needles, anxiety, and dependence on another party to administer the medication are commonly cited problems of injections. A study published in 2001 showed that 10 out of 12 patients who missed at least one injection relied on another party to administer the injection. ${ }^{13}$ Other barriers to adherence can include forgetfulness and pill burden for oral agents and interference with daily activities for intravenous agents..$^{14,15}$ In addition to these concerns, patients may perceive their treatment as unbeneficial. Many MS patients have intermittent symptoms and may not attribute their wellness to the treatment, thus failing to acknowledge the benefits of treatment. Alternatively, if symptoms begin to manifest in the patient, they may also believe the medication is failing. Unrealistic treatment expectations are a key factor of nonadherence in patients with chronic diseases. ${ }^{12}$ Side effects can also contribute to a patient's decision to discontinue therapy. Many medications used to treat MS may produce adverse events such as flu-like symptoms, depression, injection site reactions, flushing, diarrhea, or headaches. These side effects may become intolerable, decreasing a patient's compliance to the medication regimen. Other factors influencing adherence can include, but are not limited to, treatment fatigue, cognitive deficits, and cost of treatment. ${ }^{12}$

DMTs are expensive and comprise about $65 \%$ of MS treatment costs. ${ }^{16}$ Thus, clinicians, payers, and caregivers have a vested interest in identifying factors associated with adherence. Literature assessing MS disease severity and its association with DMT adherence is limited. Early studies evaluating barriers to adherence or predictors of nonadherence identified factors such as a progressive course of MS, higher disability, lower self-efficacy, lower motivation to change, and lower perceived benefits as contributors to nonadherence. ${ }^{17,18}$ In 2016, a study explored the impact of age, sex, health plan, type of DMT, and history of depression on adherence. ${ }^{19}$ Age, sex, DMT type, and depression diagnosis were determined to have a statistically significant difference in adherence to DMTs. However, none of these studies considered MS disease severity in their analysis. Other studies exploring adherence to oral DMTs did account for MS disease severity; however, the association between MS disease severity and adherence was not evaluated. ${ }^{20,21}$ To the best of our knowledge, few studies, if any, exist that explore the relationship between MS disease severity and DMT adherence. Although increasing adherence to DMTs is associated with better clinical outcomes, such as fewer MS comorbidities, relapses, hospitalizations and healthcare utilization, the factor driving this relationship has not been identified and cannot be assumed. ${ }^{22,23}$

This study explored the association of various clinical and demographic variables on DMT adherence and provides insight into the adherence of a specific population (Medicare).

\section{Methods}

\section{DATA SOURCE AND PATIENT POPULATION}

This retrospective database study used Humana Medicare Advantage claims from January 1, 2013 to December 31, 2015. The Humana dataset includes Medicare claims for both medical (inpatient and outpatient) services and prescriptions.

Medicare patients over the age of 18 with 12 months of continuous enrollment and who met the definition of MS status were included in this study. MS status was defined as at least one MS outpatient claim (ICD-9: 340, ICD-10: G35) and at least one claim for a disease modifying therapy (DMT) no more than 180 days prior to the index date. The index date was defined such that the most recent 12 months of data were used. The study period refers to the 12 months following the index date. Patients who did not use a DMT or switched DMT types during the study period were excluded from the study. After all other exclusions, removing patients that switched DMT types during the study period reduced the study population by 258 , or $7.2 \%$ (Figure 1). Patients that switched DMTs within a DMT type classification (eg, interferon beta-1a to glatiramer acetate) were not excluded by this criterion.

MS relapse was defined as an inpatient claim (hospitalization) with a listed principal diagnosis of MS or a claim for an outpatient visit with a listed diagnosis of MS in combination with intravenous or oral steroid use within 7 days. If multiple claims were present within a 30-day window, this was treated as a single relapse event, and the first available service date within such a grouping was deemed to be the relapse date..$^{24}$ 


\section{FIGURE 1 Flow Chart for Study Inclusion}

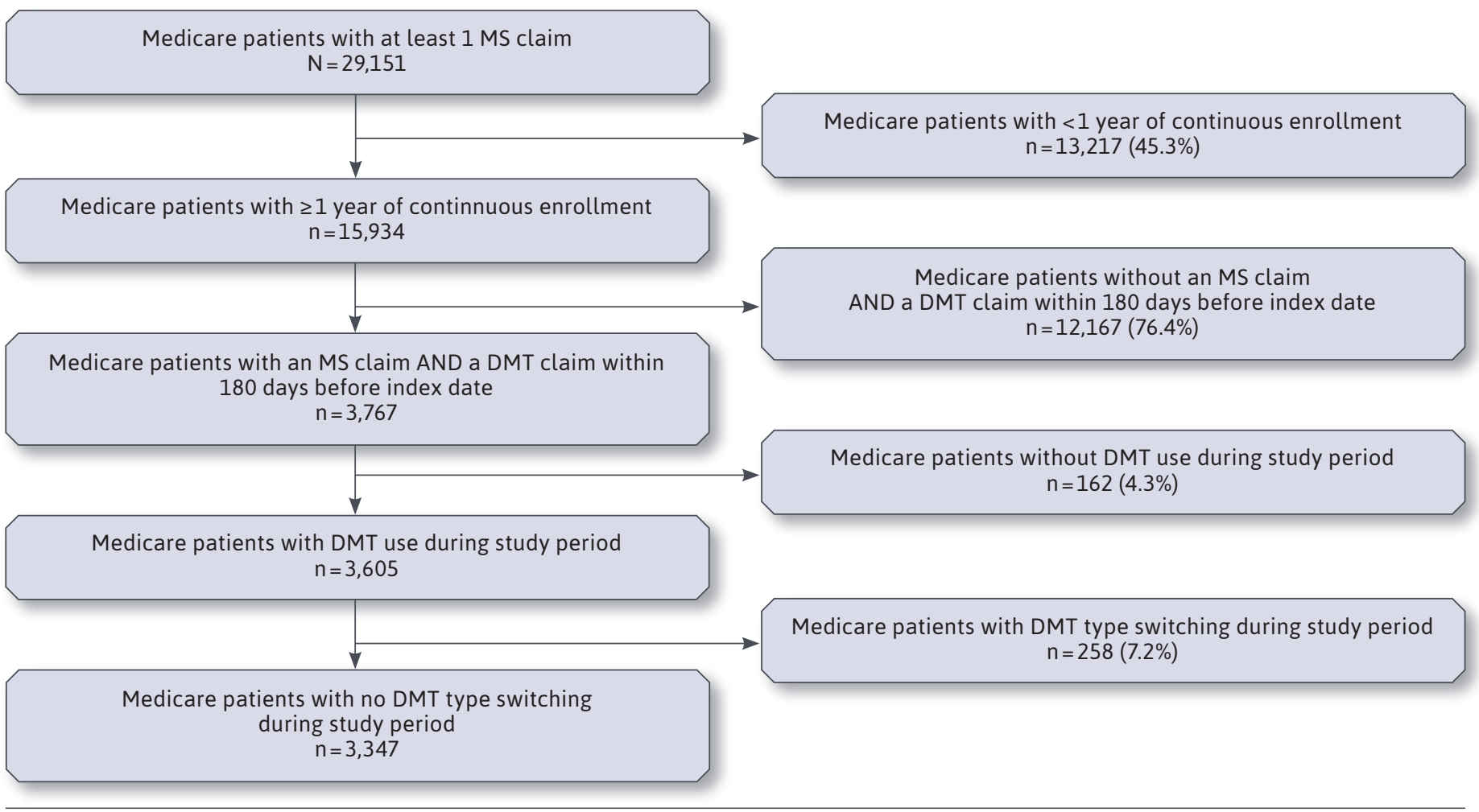

MS = multiple sclerosis, DMT = Disease-Modifying Therapy .

\section{MULTIPLE SCLEROSIS DISEASE SEVERITY}

MS disease severity in this study was derived from an algorithm that utilized summed weighted predictors in the study period. ${ }^{25-27}$ The algorithm used the binary presences of MS symptoms, disease modifying therapy (DMT) utilization, durable medical equipment (DME) utilization, MS-related hospitalizations, and MS relapses as MS disease severity predictors. DME included canes, walkers, wheelchairs, and hospital beds. A full list of MS symptoms, DME equipment, and the affiliated ICD-9, ICD-10, and CPT codes present in the database studied are available (Supplementary Table 1 and Supplementary Table 2, available in online article.) Based on the previously developed algorithm, MS disease severity predictors present in the baseline period were assigned the following weights: MS relapses or MS-related hospitalization and intravenous (IV) DMT use (natalizumab; weight=3); cerebellar movement, cerebellar walking problems, bladder/bowel symptoms, and pyramidal symptoms (weight=2); and eye symptoms, brainstem symptoms, speech symptoms, sensory symptoms, cognitive impairment, fatigue,
DME use, oral DMT use (dimethyl fumarate, fingolimod, teriflunomide), and platform DMT use (interferon beta-1a, peginterferon beta-1a, interferon beta-1b, glatiramer acetate; weight=1). For example, patients with multiple relapses received the same weight of 3 as a patient with a singular relapse. The sum of the assigned weights for each patient provided an MS disease severity score. MS disease severity scores were divided into low (score 0-4), moderate (5-10) and high $(\geq 11)$ severity categories. Although the algorithm has not been validated using the Expanded Disability Status Scale (EDSS), it has been shown to be strongly correlated with total healthcare cost and effective in estimating risk of relapse and MS-related hospitalizations. ${ }^{25-27}$ Thus, there is a reasonable expectation of accuracy.

\section{MULTIPLE SCLEROSIS ADHERENCE}

The primary outcome of interest was adherence to any DMT. Adherence to DMT was assessed using medication possession ratio (MPR) calculations. Adherence was measured during the year long (365 days) study period, and the 


\begin{tabular}{|c|c|c|c|c|}
\hline Type & Generic name & $\begin{array}{l}\text { Brand } \\
\text { name }\end{array}$ & Dosing & $\begin{array}{c}\text { Days' supply } \\
\text { (per dose) }\end{array}$ \\
\hline \multirow{6}{*}{ Platform } & interferon beta-1a & Avonex & Once weekly & 7 \\
\hline & interferon beta-1a & Rebif & Thrice weekly & 2 \\
\hline & peginterferon beta-1a & Plegridy & Every two weeks & 14 \\
\hline & interferon beta-1b & Betaseron & Every other day & 2 \\
\hline & \multirow{2}{*}{ glatiramer acetate } & \multirow{2}{*}{ Copaxone } & 20 mg: once daily & 1 \\
\hline & & & $40 \mathrm{mg}$ : thrice weekly & 2 \\
\hline \multirow{3}{*}{ Oral } & fingolimod & Gilenya & Once daily & 1 \\
\hline & teriflunomide & Aubagio & Once daily & 1 \\
\hline & dimethyl fumarate & Tecfidera & Once daily & 1 \\
\hline Intravenous (IV) & natalizumab & Tysabri & Every four weeks & 28 \\
\hline
\end{tabular}

sum of days' supply was calculated by adding all the days' supplies from both prescription and medical claims provided through the year. This total number of days of therapy was then divided by 365 days in order to calculate each patient's MPR. MPR was capped at 1.0 for those with over 365 days of medication filled. Days' supply was provided for all DMT prescription claims since "days' supply" was a variable populated in the prescription files. However, for DMT utilization noted in medical claims, "days' supply" was assumed to be consistent with the Food and Drug Administration's package insert on MS dosing. In cases where dosing windows were provided, the most conservative days' supply was used. For example, a medication dosed 3 times weekly was assigned as providing 2 days' supply per dose, or 6 days' per week. Adherence was measured both as an average MPR and in a binary capacity based on a threshold MPR of greater than or equal to $80 \%$. A sensitivity analysis was performed for MPR thresholds of greater than or equal to $70 \%$ and $90 \%$.

\section{TYPE OF DMT}

This study examined adherence to different types of DMTs. Eight different DMTs were identified and categorized intro three groups: platform (interferon beta-1a, interferon beta-1b, peginterferon beta-1a, glatiramer acetate), oral (dimethyl fumarate, fingolimod, teriflunomide), and IV (natalizumab). Table 1 summarizes the categorization of DMTs, dosing, and days' supply.

\section{STATISTICAL TESTS}

Comparisons of a binary measure of adherence (adherent vs non-adherent) for patients were compared for age category (Mann-Whitney U), gender (chi-square), DMT type (chi-square), and MS disease severity (MannWhitney U). Mann-Whitney U was considered appropriate to compare two independent groups of nominal (adherent vs non-adherent) and ordinal (low, moderate, high) data. In addition, a binary measure of adherence for patients was used to calculate the odds ratio for these various predictors using logistic regression. A sensitivity analysis for collinearity between DMT type and MS disease severity was performed by executing the multivariable logistic regression with and without DMT type as a variable. An a priori level of $\mathrm{P}<0.05$ was used to determine statistical significance. The total number of tests performed was deemed low enough such that no correction was applied for multiple testing.

\section{Results}

Data from 3,347 patients were identified and included in the analysis (Figure 1). Demographic and clinical baseline characteristics for eligible MS patients are shown in Table 2. Of those included in the study, $65.6 \%$ and $17.2 \%$ were in the age categories of 46-64 and $>65$, respectively. Participants were primarily female $(77.4 \%)$, white (78.7\%), and geographically located in the American South (61.4\%).

During the study period, 31.5\%, $58.9 \%$, and $9.6 \%$ used oral, platform, and IV DMTs, respectively. In the study population during the study period, $7.6 \%$ of patients experienced at least one MS-related hospitalization and $20.0 \%$ experienced at least one MS relapse.

The most prevalent baseline MS-related symptoms were fatigue (36.3\%) and cerebellar walking problems (32.8\%), followed by bladder symptoms (25.7\%), pyramidal symptoms (21.5\%), cerebellar movement problems (18.6\%), brainstem symptoms (18.3\%), sensory symptoms (14.7\%), cerebral cognitive symptoms (12.2\%), and eye symptoms (9.4\%; See Table 2). In addition, $8.7 \%$ of the sample used durable medical equipment.

Table 3 outlines the MPR and adherence for each category investigated. The study population had an average MPR of 84.7 with $75 \%$ being adherent. Significant differences were detected between groups for MS disease severity $(P<0.001)$ and age category $(\mathrm{P}<0.001)$. The percentage of adherent patients was $77 \%, 75 \%$, 


\section{TABLE 2 Baseline Demographics, Utilization, and Disease Severity}

\begin{tabular}{|c|c|c|c|c|c|}
\hline Overall $(\mathrm{N}=3,347)$ & Mean & $\begin{array}{l}\text { Standard } \\
\text { deviation }\end{array}$ & & $\begin{array}{c}\text { Number of } \\
\text { patients }\end{array}$ & $\begin{array}{c}\text { Percentage } \\
(\%)\end{array}$ \\
\hline Medication possession ratio (MPR) & 0.85 & & \multicolumn{3}{|l|}{ Multiple sclerosis disease severity } \\
\hline Percent adherence (MPR $>0.8), \%$ & 75 & & $\operatorname{Low}(0-4)$ & 1,868 & 55.8 \\
\hline \multirow[t]{2}{*}{ Age (years) } & 54.9 & 9.7 & Moderate (5-10) & 1,212 & 36.2 \\
\hline & $\begin{array}{c}\text { Number of } \\
\text { patients }\end{array}$ & Percentage & High $(\geq 11)$ & 267 & 8.0 \\
\hline \multicolumn{3}{|l|}{ Age category, years } & \multicolumn{3}{|l|}{ DMT type during study period } \\
\hline $18-45$ & 576 & 17.2 & Oral & 1,055 & 31.5 \\
\hline $46-64$ & 2,196 & 65.6 & Platform & 1,971 & 58.9 \\
\hline$\geq 65$ & 575 & 17.2 & IV & 321 & 9.6 \\
\hline \multicolumn{3}{|l|}{ Gender } & \multicolumn{3}{|l|}{ Acute exacerbations during baseline } \\
\hline Male & 757 & 22.6 & $>1$ Hospitalizations & 254 & 7.6 \\
\hline Female & 2,590 & 77.4 & $>1$ Relapses & 671 & 20.0 \\
\hline \multicolumn{3}{|l|}{ Race } & \multicolumn{3}{|c|}{ Symptoms experienced during baseline } \\
\hline White & 2,634 & 78.7 & Eye & 314 & 9.4 \\
\hline Black & 570 & 17.0 & Brainstem & 612 & 18.3 \\
\hline Asian & 10 & 0.3 & Cerebellar-walking & 1,097 & 32.8 \\
\hline Hispanic & 51 & 1.5 & Cerebellar-movement & 623 & 18.6 \\
\hline North american native american & 4 & 0.1 & Pyramidal & 720 & 21.5 \\
\hline \multicolumn{3}{|l|}{ Geographical region } & Sensory & 493 & 14.7 \\
\hline Midwest & 78 & 23.3 & Bladder & 861 & 25.7 \\
\hline Northeast & 121 & 3.6 & Cerebral cognitive & 410 & 12.2 \\
\hline South & 2,054 & 61.4 & Fatigue & 1,215 & 36.3 \\
\hline West & 391 & 11.7 & Durable medical equipment use & 291 & 8.7 \\
\hline
\end{tabular}

$D M T=$ Disease Modifying Therapy; $I Q R=$ interquartile range; $M P R=$ medication possession ratio.

and $61 \%$ for low, moderate, and high MS disease severity, respectively. Analysis of adherence by age category demonstrated the highest percentage adherence in patients 65 or older $(79 \%)$. The age category with the lowest percentage adherent was 18-45 (70\%) with 46-64 in between (76\%). No difference was seen in adherence levels between genders $(\mathrm{P}=0.948)$ or DMT type $(\mathrm{P}=0.273)$. The sensitivity analysis for MPR threshold demonstrated minimal fluctuation and did not impact results.

Multivariable logistic analysis demonstrated that the 46-64 and >65 age groups were 1.33 (OR: 1.33 [95\% CI 1.08-1.64]) and 1.55 (OR: 1.55, [95\% CI 1.18-2.05]) times more likely to be adherent compared to the 18-45 age group. Those with high MS disease severity were less likely to be adherent than their low MS disease severity counterparts (OR: 0.47, [95\% CI 0.36-0.62]). Finally, those with moderate MS disease severity were $10 \%$ less likely to be adherent compared to those with low MS disease severity although this difference was not statistically significant. Results of the multivariable logistic analysis are summarized in Table 4 . The analysis was performed with MPR thresholds of $70 \%, 80 \%$, and $90 \%$, as well as with and without DMT type as a variable. The results were not sensitive to MPR threshold or collinearity between DMT type and MS disease severity.

\section{Discussion}

To the best of our knowledge, this study is one of the first to explore the relationship between MS disease severity and DMT adherence. Given the large proportion of US patients with MS enrolled in Medicare (25-30\%), ${ }^{28-30}$ this study provides valuable insight into this population. The average age was similar to those identified in previous studies, as was 


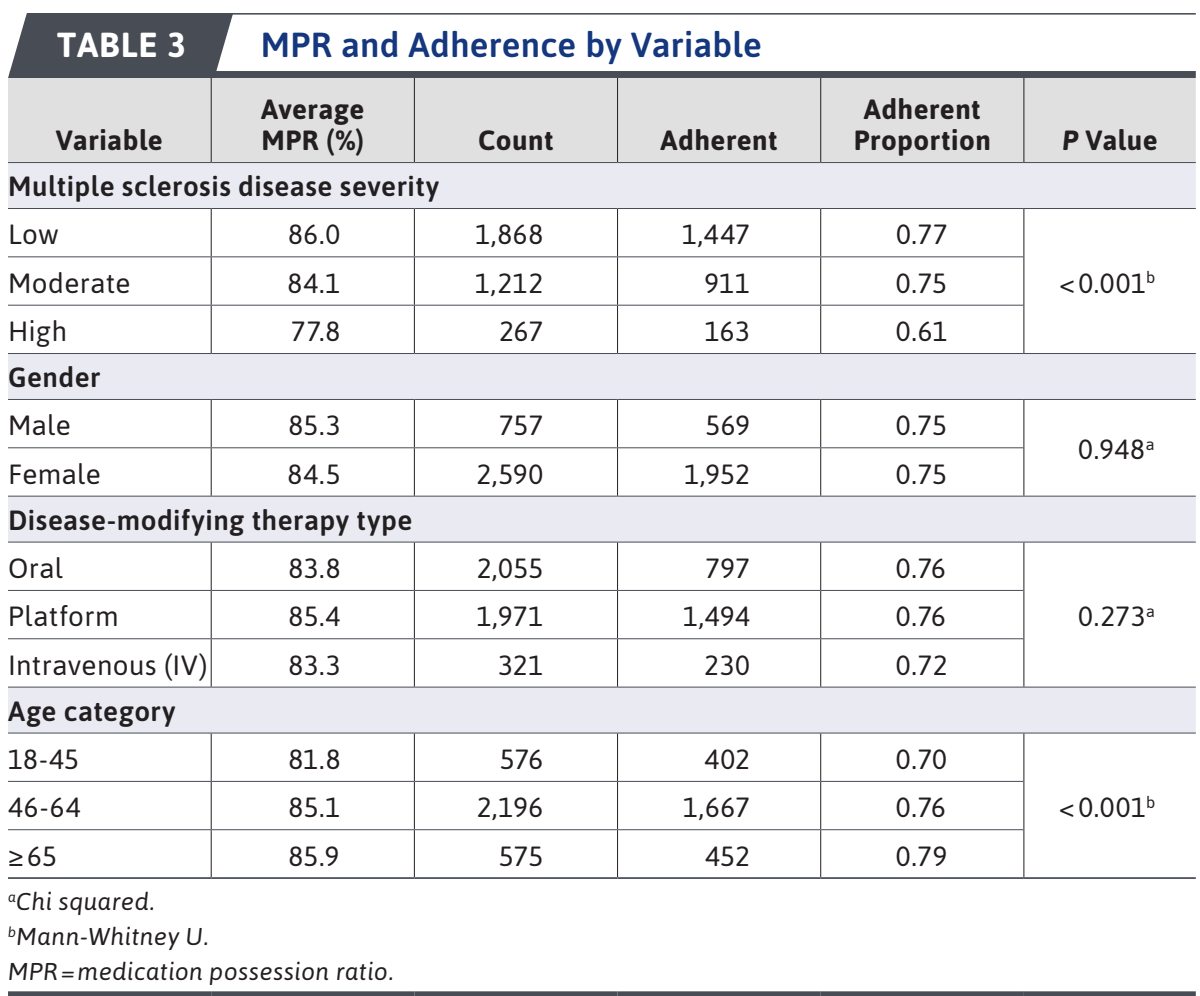

the proportion of the population identifying as female. ${ }^{19,31}$ A recent study in 2020 demonstrated similar racial demographics. ${ }^{31}$ Of potential significance is the difference in number of relapses. Whereas we report $20 \%$ having $>1$ relapse, the aforementioned study reported a slightly higher value of $26 \% .^{31}$ This may be explained by regional differences in the sample population. With more than $60 \%$ of our participants identified as living in the South, relapses may be lower given the association between latitude and MS incidence. ${ }^{19,31-34}$ Another variable that could contribute to this difference is the type of MS, such as relapsing-remitting, primary progressive, and secondary progressive MS, although MS type was not captured in the claims data.

Our results indicated a higher average MPR and rate of adherence than would typically be expected in the general population. We expect this difference is related to the population studied, Medicare patients, which agrees with comparisons between studies specific to commercial plans and those including Medicare patients..$^{19,35}$ Medication adherence is a large component of Medicare Star Ratings and provides one possible explanation for the higher overall adherence rates generally seen in Medicare plans. Additionally, about $83 \%$ of our population was less than 65 years of age. It is reasonable to assume the majority of MS patients under the age of 65 on Medicare were enrolled due to disability since the only criteria for Medicare eligibility under age 65 is end-stage renal disease, amyotropic lateral sclerosis, or at least 24 months of enrollment in Social Security Disability benefits. A previous study demonstrated an association between disability and higher adherence which might explain the higher average MPR and rate of adherence found in our results. ${ }^{31}$

Statistical analyses demonstrated similar findings in multiple studies regarding adherence and age, with higher adherence being associated with increased age. ${ }^{19,36,37}$ This may be related to increased awareness of health status with older age; ${ }^{38}$ however, the association between older age and adherence is not well understood ${ }^{37}$ Sex was not determined to be associated with adherence. Review of the literature indicates inconclusive findings regarding sex, with some reporting an association between men and increased adherence, albeit a relatively small increase. ${ }^{7,19,39}$

DMT type was not associated with significant differences in adherence, although the trends appear to indicate better adherence with platform and oral DMTs. This agrees with other literature which also determined DMT type was not a statistically significant predictor of adherence; however, the studies referenced were not specific to Medicare patients. ${ }^{19,40}$ In contrast, some studies have shown the oral DMT fingolimod is associated with better adherence compared to injectable DMTs. ${ }^{21,41-43}$ Compared to IV and platform DMTs, oral DMTs benefit from convenient dosing intervals (once daily or twice daily) and does not involve needles. More research is needed to further evaluate the impact of newer oral DMTs and adherence.

The results indicate a significant difference in adherence exists between those with low MS disease severity and high MS disease severity. In other words, the severity of MS is associated with adherence. While patient factors (such as age, race, ethnicity, and sex) and medication factors (such as dosage form, route of administration, and side effects) are frequently considered 


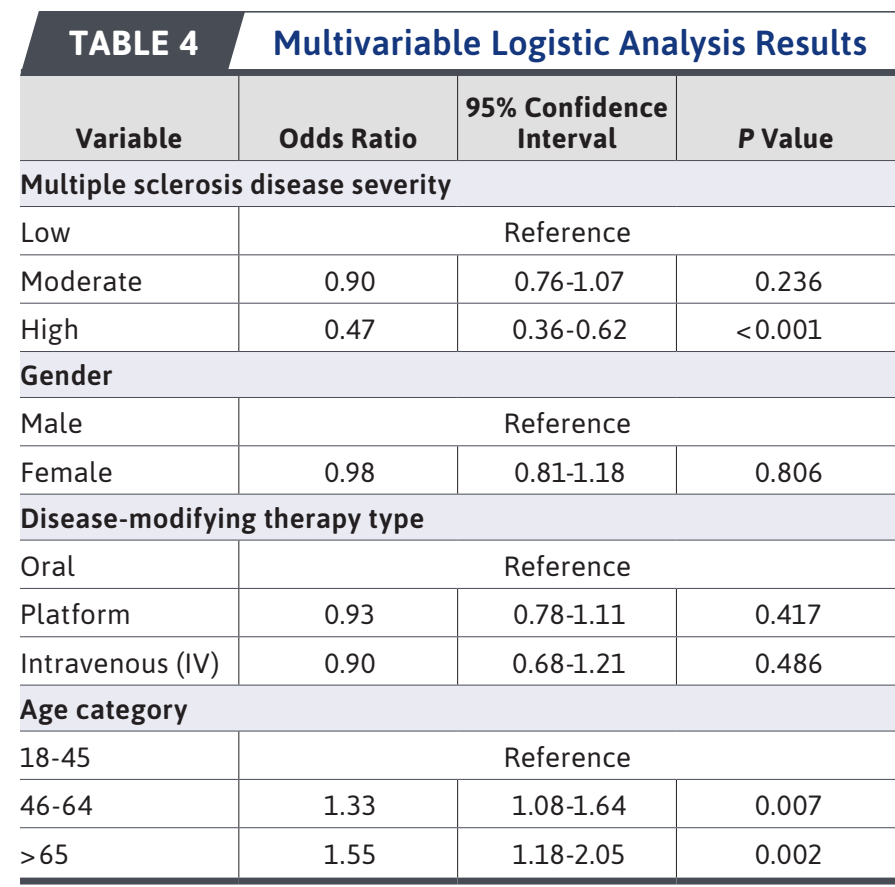

when assessing risk of low adherence, the impact of disease severity is often neglected. Our study found that patients with a high MS disease severity score were $53 \%$ less likely to be adherent compared to the low MS disease severity group. Additionally, only $61 \%$ of patients in the high MS disease severity category met the criteria for adherence. This falls closer to average adherence rates for more common chronic disease states like hypercholesterolemia, osteoporosis, and type 2 diabetes. ${ }^{44}$ This study indicates that MS disease severity is another important consideration when evaluating risk of low adherence.

While clinicians benefit from in-person patient visits and access to electronic health records to assess individual adherence risk factors, other decision makers are limited to claims data alone. This presents a challenge to various managed care plans seeking targeted interventions to improve health outcomes and mitigate costs at the population level. This study identifies a factor not yet considered in claims-based algorithms to identify MS patients at high risk of low adherence. By identifying discrete factors associated with low adherence that are accessible using claims data, decision makers can use those factors to tailor their adherence optimization strategies. For example, an MS disease severity score of $>11$ could be considered alongside factors such as age to identify which plan members would benefit from targeted calls and mail campaigns designed to improve adherence.

\section{LIMITATIONS}

The study is subject to limitations common to retrospective claims-based studies. These data do not differentiate between categories of MS such as relapsing-remitting MS or primary-progressive MS, which impact both drug selection and MS disease severity. This study does not include more recently approved MS DMTs such as cladribine (Mavenclad), ocrelizumab (Ocrevus), and ozanimod (Zeposia). This may decrease generalizability of results to present day. Additionally, claims-based adherence using MPR is an indirect measurement and cannot confirm actual medication use. Despite this limitation, claims-based adherence is commonly seen in the literature as a proxy for adherence. ${ }^{19,31}$

This study may not translate to commercially insured patients as adherence is generally lower in these populations. Although studies demonstrate consistency in some relationships between adherence and factors like age and DMT type when comparing Medicare and commercial populations, this cannot be assumed for adherence and MS disease severity. We also recognize that 258 patients were excluded from this study for switching DMT types during the study period. This may further limit generalizability although only $7.2 \%$ of the population was removed by this exclusion. This was also performed to specifically examine patients assumed to be stable on therapy.

In addition, the days' supply for DMTs in medical files had to be estimated. Although these values were based on FDA dosing guidelines, package inserts, and previous studies with the most conservative ones being used in the final analysis, underestimation of days' supply is possible.

Finally, this study looked at the association between MS disease severity and adherence, not causation. Other variables, such as length of time with MS and previous treatment patterns were not included in the database. These variables may have been associated with treatment resistance to new therapies, which in turn might decrease adherence rates.

\section{Conclusions}

Adherence to DMTs in MS is associated with increased age and low MS disease severity. MS disease severity should be considered when evaluating risk of low adherence, in addition to patient and medication factors. Further analysis is warranted to consider newer agents, side-effect profiles of medications, and past treatment patterns. Future research should also be directed toward measuring the effects of targeted interventions in those populations demonstrating the lowest adherence rates. 


\section{DISCLOSURES}

No funding supported this project. The authors have nothing to disclose.

Preliminary results were previously presented virtually at AMCP Annual 2020 in April 2020.

\section{REFERENCES}

1. Galetta KM, Bhattacharyya S. Multiple sclerosis and autoimmune neurology of the central nervous system. Med Clin North Am. 2019 Mar 1;103(2):325-36.

2. Rae-Grant A, Day GS, Marrie RA, et al. Practice guideline recommendations summary: Disease-modifying therapies for adults with mutliple sclerosis. Report of the Guideline development, Disemmination, and Implementation Subcommittee of the American Academy of Neurology. Neurology. 2018;90(17):777-88.

3. Ebers GC. Randomised doubleblind placebo-controlled study of interferon $\beta$-1a in relapsing/remitting multiple sclerosis. Lancet. 1998 Nov 7;352(9139):1498-504.

4. Jacobs LD, Cookfair DL, Rudick RA, et al. Intramuscular interferon beta1a for disease progression in relapsing multiple sclerosis. Ann Neurol. 1996 Mar;39(3):285-94.

5. Río J, Comabella M, Montalban X. Multiple sclerosis: current treatment algorithms. Curr Opin Neurol. 2011 Jun 1;24(3):230-37.

6. Adherence to Long-Term Therapies: Evidence for Action. World Health Organization; 2003. https://apps. who.int/iris/bitstream/handle/10665/42682/9241545992.pdf

7. Ivanova JI, Bergman RE, Birnbaum HG, Phillips AL, Stewart M, Meletiche DM. Impact of medication adherence to disease-modifying drugs on severe relapse, and direct and indirect costs among employees with multiple sclerosis in the US. J Med Econ. 2012;15(3):601-09.

8. Simpson SH, Eurich DT, Majumdar SR, et al. A meta-analysis of the association between adherence to drug therapy and mortality. BMJ. 2006;333(7557):15.
9. Sokol MC, McGuigan KA, Verbrugge RR, Epstein RS. Impact of Medication Adherence on Hospitalization Risk and Healthcare Cost. Med Care. 2005;43(6):521-30.

10. Steinberg SC, Faris RJ, Chang CF, Chan A, Tankersley MA. Impact of Adherence to Interferons in the Treatment of Multiple Sclerosis. Clin Drug Investig. 2010;30(2):89-100.

11. Tan H, Cai Q, Agarwal S, Stephenson JJ, Kamat S. Impact of adherence to disease-modifying therapies on clinical and economic outcomes among patients with multiple sclerosis. Adv Ther. 2011;28(1):51-61.

12. Costello K, Kennedy P, Scanzillo J. Recognizing nonadherence in patients with multiple sclerosis and maintaining treatment adherence in the long term. Medscape J Med. 2008;10(9):225.

13. Goodkin DE, Boudewyn AC, Mohr DC, et al. Injectable medication for the treatment of multiple sclerosis: The influence of self-efficacy expectations and injection anxiety on adherence and ability to selfinject. Ann Behav Med. 2001;23(2):125-32.

14. Aungst A, Casady L, Dixon C, et al. Assessing Barriers to Adherence with the Use of Dimethyl Fumarate in Multiple Sclerosis. Clin Drug Investig. 2020 Jan 1;40(1):73-81.

15. Kołtuniuk A, Rosińczuk J. Adherence to disease-modifying therapies in patients with multiple sclerosis. Patient Prefer Adherence. 2018;12:1557.

16. Earla JR, Thornton JD, Hutton GJ, et al. Marginal Health Care Expenditure Burden Among U.S. Civilian Noninstitutionalized Individuals with Multiple Sclerosis. J Manag Care Spec Pharm. 2020;26(6):741-49.

17. Patti F. Optimizing the benefit of multiple sclerosis therapy: the importance of treatment adherence. Patient Prefer Adherence. 2010;4:1.

18. Klauer T, Zettl UK. Compliance, adherence, and the treatment of multiple sclerosis. J Neurol. 2008 Dec 1;255(6):87-92.
19. Higuera L, Carlin CS, Anderson S. Adherence to Disease-Modifying Therapies for Multiple Sclerosis. J Manag Care Spec Pharm. 2016;22(12):1394-401.

20. Ontaneda D, Nicholas J, Carraro M, et al. Comparative effectiveness of dimethyl fumarate versus fingolimod and teriflunomide among MS patients switching from first-generation platform therapies in the US. Mult Scler Relat Dis. 2019 Jan 1;27:101-11.

21. Earla JR, Hutton GJ, Thornton JD, et al. Comparative Adherence Trajectories of Oral Fingolimod and Injectable Disease Modifying Agents in Multiple Sclerosis. Patient Prefer Adherence. 2020;14:2187.

22. Burks J, Marshall TS, Ye X. Adherence to disease-modifying therapies and its impact on relapse, health resource utilization, and costs among patients with multiple sclerosis. Clinicoecon Outcomes Res. 2017;9:251.

23. Lizán L, Comellas M, Paz S, et al. Treatment adherence and other patientreported outcomes as cost determinants in multiple sclerosis: a review of the literature. Patient Prefer Adherence. 2014;8:1653.

24. Chastek BJ, Oleen-Burkey M, Lopez-Bresnahan MV. Medical chart validation of an algorithm for identifying multiple sclerosis relapse in healthcare claims. J Med Econ. 2010;13(4):618-25. doi: 10.3111/13696998.2010.523670

25. Nicholas J, Ontaneda D, Carraro M, et al. Development of an Algorithm to Identify Multiple Sclerosis (MS) Disease Severity Based on Healthcare Costs in a US Administrative Claims Database. In: 2017.

26. Toliver J, Barner JC, Lawson K, et al. Replication of a Claims-based Algorithm to Estimate Multiple Sclerosis Disease Severity in a Commercially Insured Population. Mult Scler Relat Disord. 2020 Sep 28:102539.

27. Toliver JC, Barner JC, Lawson KA, et al. Use of a Claims-based Algorithm to Estimate Disease Severity in the Multiple Sclerosis Medicare Population. Mult Scler Relat Disord. 2021 Jan 6:102741. 
28. Owens GM. Economic burden of multiple sclerosis and the role of managed sare organizations in multiple sclerosis management. Am J Manag Care. 2016 Jun;22(6 Suppl):s151-58.

29. Mathis AS, Owens GM. Implications for multiple sclerosis in the era of the Affordable Care Act: the shifting managed care landscape. Am J Manag Care. 2014 Dec 1;20(11 Suppl):S242-53.

30. National Multiple Sclerosis Society. Medicare. Accessed May 10, 2020. https://www.nationalmssociety.org/ Living-Well-With-MS/Work-and-Home/ Insurance-and-Financial-Information/ Health-Insurance/Medicare

31. Li P, Ladage VP, Berger J, et al. Disease-Modifying Therapy Adherence and Associated Factors in a National Sample of Medicare Patients With Multiple Sclerosis. Value Health. 2020;23(3):328-34.

32. Tremlett H, Van Der Mei IA, Pittas F, et al. Monthly ambient sunlight, infections and relapse rates in multiple sclerosis. Neuroepidemiology. 2008;31(4):271-79.

33. Kamel FO. Factors involved in relapse of multiple sclerosis. J Microsc Ultrastruct. 2019 Jul;7(3):103.

34. Spelman T, Gray O, Trojano M, et al. Seasonal variation of relapse rate in multiple sclerosis is latitude dependent. Ann Neurol. 2014 Dec;76(6):880-90.
35. Halpern R, Agarwal S, Dembek C, Borton L, Lopez-Bresnahan M. Comparison of adherence and persistence among multiple sclerosis patients treated with disease-modifying therapies: a retrospective administrative claims analysis. Patient Prefer Adherence. 2011;5:73-84.

36. Verdugo RM, Herráiz ER, Fernández-Del Olmo R, Bonet MR, García MV. Adherence to disease-modifying treatments in patients with multiple sclerosis in Spain. Patient Prefer Adherence. 2019;13:261.

37. Lahdenperä S, Soilu-Hänninen M, Kuusisto HM, Atula S, Junnila J, Berglund A. Medication adherence/ persistence among patients with active multiple sclerosis in Finland. Acta Neurol Scand. 2020 Dec;142(6):605-12.

38. Kim SJ, Kwon OD, Han EB, et al. Impact of number of medications and age on adherence to antihypertensive medications: A nationwide population-based study. Medicine. 2019 Dec;98(49).

39. Halpern R, Agarwal S, Borton L, Oneacre K, Lopez-Bresnahan MV. Adherence and persistence among multiple sclerosis patients after one immunomodulatory therapy failure: retrospective claims analysis. Adv Ther. 2011 Sep 1;28(9):761.
40. Munsell M, Frean M, Menzin J, Phillips AL. An evaluation of adherence in patients with multiple sclerosis newly initiating treatment with a self-injectable or an oral disease-modifying drug. Patient Prefer Adherence. 2017;11:55.

41. Williams MJ, Johnson K, Trenz HM, et al. Adherence, persistence, and discontinuation among Hispanic and African American patients with multiple sclerosis treated with fingolimod or glatiramer acetate. Curr Med Res Opin. 2018 Jan 2;34(1):107-15.

42. Agashivala N, Wu N, Abouzaid S, et al. Compliance to fingolimod and other disease modifying treatments in multiple sclerosis patients, a retrospective cohort study. BMC Neurol. 2013;13:9.

43. Bergvall N, Petrilla AA, Karkare SU, et al. Persistence with and adherence to fingolimod compared with other disease modifying therapies for the treatment of multiple sclerosis: a retrospective US claims database analysis. J Med Econ. 2014;17(10):696-707.

44. Briesacher BA, Andrade SE, Fouayzi H, Chan KA. Comparison of drug adherence rates among patients with seven different medical conditions. Pharmacotherapy. 2008 Apr;28(4):437-43. 